\title{
Adaptive Random Link PSO with Link Change Variations and Confinement Handling
}

\author{
Mrs. Snehal Mohan Kamalapur \\ Research Scholar, Dr. D Y Patil Institute of Engineering and Technology, Pune, University of Pune, Maharashtra, India \\ E-mail:snehal_kamalapur@yahoo.com \\ Dr. Varsha Hemant Patil \\ Research Guide, University of Pune, Maharashtra, India \\ E-mail: varsha.patil@gmail.com
}

\begin{abstract}
Particle Swarm Optimization is swarm based optimization technique. Swarm consists of particles and the particles fly through the problem space in Particle Swarm Optimization (PSO). Confinement methods and parameters such as Inertia Weight, Neighborhood of the particle have major impact on PSO performance. The paper presents variations of the PSO with adaptive random link neighborhood. The work carried out considers linearly decreasing inertia weight and different confinement methods. The performance of adaptive random link PSO by geometrical updation of velocity with confinement methods is tested here.
\end{abstract}

Index Terms - Adaptive Random Link, Confinement, Inertia Weight, Neighborhood, SPSO

\section{INTRODUCTION}

Particle Swarm Optimization (PSO) is an optimization technique developed by Dr. Eberhart and Dr. Kennedy in 1995 [1][2][3]. The swarm is analogous to social interaction. The particle in swarm is a learner as well as a guide. Every particle interacts and changes its position dynamically. The particles fly through problem space. A particle can be repositioned or its velocity can be modified to prevent it from leaving the search space. The repositioning of particle is done by using position confinement approach. Velocity of the particle is restricted in search space by using different velocity confinement methods.

Computational behavior of PSO is affected by parameter modification. Parameters include swarm size, acceleration coefficient and inertia weight or constriction coefficient. The parameters of the PSO are revised by parameter modification. Parameter setting is important as the performance of PSO is sensitive to the parameter settings.

Section II explains particle swarm optimization while section III and IV describe highlights on confinement methods and adaptation. Parameter control and Benchmark functions are briefly described in section $\mathrm{V}$ and VI respectively. Section VII focuses on experimental set up. Section VIII experimentally validates the correlation. The paper closes with conclusions in section IX.

\section{PARTICle SWARM Optimization}

Particle Swarm Optimization is swarm or population based optimization technique. The first version of the PSO was published by Kennedy and Eberhart in 1995 [1][2] and it has rapidly progressed in recent years since then. The particles move iteratively within the search space. Two main versions of PSO namely Global Best version and Local best version were introduced in early phase of PSO research. Global best is the best position achieved by any of the particle in the swarm. Global best particle is influential and influences to update position of all particles. Local best is the best position achieved in the neighborhood of a particle.

Swarm of N particles is represented as-

$$
\mathrm{S}=\left\{x_{1}, x_{2}, \ldots, x_{N}\right\}
$$

For a D-dimensional search space, the position of the $\mathrm{i}^{\text {th }}$ particle is represented as:

$$
x_{i}=\left(x_{i l}, x_{i 2}, \ldots, x_{i D}\right)^{\mathrm{T}} \quad \text { where } \mathrm{i}=1,2, \ldots, \mathrm{N}
$$

Let $\mathrm{t}$ denote iteration counter. So $x_{i}(t)$ and $v_{i}(t)$ denote current position and velocity of $\mathrm{i}^{\text {th }}$ particle respectively.

The position of each particle is updated using a proper position shift. This is called velocity and is denoted as

$$
v_{i}=\left(v_{i l}, v_{i 2}, \ldots, v_{i D}\right)^{\mathrm{T}} \quad \text { where } \mathrm{i}=1,2, \ldots, \mathrm{N}
$$

\section{A. Algorithm of Global Best version of PSO}

- Initialize the population of particles randomly Do

- Calculate fitness values of each particle as per objective function

- Update particle's best position if the current fitness value is better than earlier best position

- Determine the best fitness value in the swarm

- Update velocity of each particle using (1)

$$
\begin{aligned}
& v_{i}(t+1)=v_{i}(t)+c_{1} * r_{1} *\left(p_{i}-x_{i}\right)+c_{2} * r_{2} * \\
& \left(p_{g}-x_{i}\right)
\end{aligned}
$$

- Update position of particle using equation (2)

$$
x_{i}(t+1)=x_{i}(t)+v_{i}(t+1)
$$

While maximum number of iterations or criterion is not attained 


\section{B. Algorithm of Local best version of PSO}

- Initialize the population of particles randomly Do

- Calculate fitness values of each particle as per objective function

- Update particle's best position if the current fitness value is better than earlier best position

- Determine the best fitness value in the swarm

- Update velocity of each particle using (3)

$$
\begin{aligned}
v_{i}(t+1)= & v_{i}(t)+c_{1} * r_{1} *\left(p_{i}-x_{i d}\right)+ \\
& c_{2} * r_{2} *\left(p_{l}-x_{i}\right)
\end{aligned}
$$

- Update position of particle using equation (2)

While maximum number of iterations or criterion is not attained

\section{Standard PSO 2011}

Three Standard PSO algorithms have been defined in the literature of PSO [4], [5], [6], [7], [8], [9]. Until 2007, the velocity update was carried out dimension by dimension method. It is known that dimension by dimension method is biased [10]. When the optimum point lies on an axis, on a diagonal or on the centre of the system of coordinates then it is easy to find it for PSO. Spear analyzed this in 2010 [11]. Clerc proposed SPSO2011 as a solution to this bias [5]. Standard PSO (SPSO-2011) [8], [9] exploits the idea of rotational invariance. SPSO-2011 modifies velocity in geometrical way.

Let $G_{i}(t)$ be the centre of gravity of the current position, a point a bit beyond the best previous position and a point a bit beyond the best previous position in the neighborhood.

Center of gravity is calculated using (4)-

$$
G_{i}(t)=x_{i}+c * \frac{p_{i}+l_{i}-2 * x_{i}}{3}
$$

Let $x_{i}$ ' be random point defined using uniform distribution in the hypersphere of radius $G_{i}$ and center $\left(G_{i^{-}} x_{i}\right)$ that is $H_{i}\left(G_{i},\left\|G_{i}-x_{i}\right\|\right)$

Velocity of the particle is then calculates using (5)

$$
v_{i}(t+1)=\omega * v_{i}(t)+x^{\prime}{ }_{i}(t)-x_{i}(t)
$$

Position of the particle is calculates using (2).

\section{PARAMETERS OF PSO}

\section{- Swarm Size :}

The Swarm size is a size of the population. The swarm consists of particles. Swarm size varies from 20 to 60 . Ideally swarm size is considered as 40 . The swarm size can also be computed using (6) -

$$
s=10+[2 * \operatorname{sqrt}(D)]
$$

\section{- Velocity Threshold}

The first issue observed and addressed by researchers is swarm explosion. Solution put forth by researcher was velocity clamping. Velocity clamping prevents particles uncontrolled increase of magnitude from current positions. Whenever velocity goes beyond threshold bound it is directly set to closest bound or threshold. Details of velocity confinement are discussed in section IV.

\section{- Inertia Weight}

Inertia weight was not used in the first version of PSO developed by Eberhart. As swarm was not able to converge towards promising position with velocity clamping, a new parameter inertia weight $\omega$ was introduced [12] in the equation (1) as shown in (7)

$$
\begin{aligned}
v_{i d}(t+1)= & \omega * v_{i d}(t)+c_{1} * r_{1} *\left(p_{i d}-x_{i d}\right)+ \\
& c_{2} * r_{2} *\left(p_{g d}-x_{i d}\right)
\end{aligned}
$$

The inertia weight defines the impact of previous velocity of each particle to the current one and controls the scope of search. Poli et al. [13] interpreted it as the fluidity of the medium in which particles move.

Many variations of PSO were proposed based on inertia weight. The Linearly Decreasing[12][14], Linearly increasing [15] , Nonlinear [16], Sigmoid decreasing [17] , Adaptive [18], Random [19], Chaotic [20], Oscillating [21], Logarithmic decreasing [22], Exponent Decreasing inertia weight strategies [23], [24], [25] and Fuzzy Adaptive Inertia Weight [26] were used in the literature. The Linearly Decreasing strategy improves the efficiency and performance of PSO.

\section{- Acceleration Coefficient}

Acceleration coefficients $c_{1}$ and $c_{2}$ indicate cognitive and social influence values respectively.

\section{- Neighborhood Topology}

Neighborhood topology is a scheme to determine the neighbors of particles in a swarm. Information exchange among the particles is related to exploration capability of the swarm. Each particle may have set of other particles as neighbors.

The actual distance of the particles can be calculated to form neighborhood. But this requires $(\mathrm{N}(\mathrm{N}+1))^{2}$ computations at each iteration, if $\mathrm{N}$ is size of the swarm. Simple alternate solution to this is to use indices of particles to decide neighborhood. The cardinality of neighborhood called neighborhood size depends on type of neighborhood topology.

In PSO, each particle has communication neighborhood so several studies were performed in order to determine effect of the neighborhood topology on the convergence.

The performance of PSO can be improved by selecting proper neighborhood topology.

The neighborhood topology can be classified as [27], [28]-

- gbest Topology

- Ring Topology

- Adaptive Random

- Mesh Topology

- Tree or hierarchical Topology

- Toroidal Topology

- Dynamic Topology 
- lbest Ring Lattice with dynamic increment neighborhood

- Fitness Distance Ratio

- Random Edge Migration

- Dynamic Hierarchy

- TRIBES

Some topologies are good for global optimization while some topologies are good for local optimization.

\section{CONFINEMENTS OF PSO}

A particle tends to leave the search space in Particle Swarm Optimization. A particle can be repositioned or velocity can be modified to prevent it from leaving the search space [29]. These two types of confinement are discussed here.

\section{A. Prevention}

Whenever velocity goes beyond threshold bound one of the following confinement is used for Velocity of Particle in the literature-

1. Absorb or Zero

$$
\begin{gathered}
\text { if } v(t+1)>v_{\max } \text { or } v(t+1)<v_{\max } \\
\text { then } v(t+1)=0
\end{gathered}
$$

2. Deterministic Back

$$
\text { if } \begin{gathered}
v(t+1)>v_{\max } \text { or } v(t+1)<v_{\max } \\
v(t+1)=-\lambda * v(t+1)
\end{gathered}
$$

Where $\boldsymbol{\lambda}$ can be a predefined value.

3. Random back

If velocity goes beyond threshold bound the velocity is reversed back by multiplying with random value drawn from uniform distribution [ $\left[\begin{array}{ll}0 & 1\end{array}\right]$

4. Adjust

If velocity goes beyond threshold bound it is adjusted using position of the particle-

$$
\mathrm{v}(\mathrm{t}+1)=\mathrm{x}(\mathrm{t}+1)-\mathrm{x}(\mathrm{t})
$$

5. Hyperbolic

If velocity goes beyond threshold bound it is normalized as

$$
\begin{aligned}
& v(t+1)=\frac{v(t+1)}{1+\left|\frac{v(t+1)}{x_{\max }-x(t)}\right|} \text { if } v(t+1)>0 \\
& v(t+1)=\frac{v(t+1)}{1+\left|\frac{v(t+1)}{x(t)-x_{\min }}\right|} \text { if } v(t+1)<0
\end{aligned}
$$

\section{B. Repositioning Particle}

Whenever position of the particle goes beyond threshold bound one of the following confinement is used

1. Nearest
When the position of the particle moves away from the boundary the particle is adjusted using (13)(14)

$$
\begin{aligned}
& \text { if } x(t+1)>x_{\text {max }} \text { then } x(t+1)=x_{\text {max }} \\
& \text { if } x(t+1)<x_{\text {min }} \text { then } x(t+1)=x_{\text {min }}
\end{aligned}
$$

2. Do not Change

When the position of the particle moves away from the boundary the particle is adjusted using (15)

$$
\text { if } \begin{aligned}
x(t+1) & >x_{\max } \text { or } x(t+1)<x_{\min } \text { then } \\
x(t+1) & =x(t+1)-v(t+1)
\end{aligned}
$$

3. Reflex

When the position of the particle moves away from the boundary the particle is adjusted using (16)(17)

$$
\begin{aligned}
& \text { if } x(t+1)>x_{\max } \text { then } x(t+1)=x_{\text {max }}- \\
& \left(x_{\text {max }}-x(t+1)\right) \\
& \quad \text { if } x(t+1)<x_{\text {min }} \\
& \left(x_{\text {min }}-x(t+1)\right)
\end{aligned}
$$

\section{PARAMETER CONTROL}

The parameter control can be used depending on how and what is changed. Parameters can be controlled using deterministic rule or by adaptation. The deterministic rule is used to control the parameter value for all iterations. This gives better performance for some cases but not in all cases. Hence the parameter control can be done using current state of the search.

Adaptation used here is based on two parameters-

\section{Inertia Weight}

The searching varies from exploratory phase, towards the refinement of local search at the end. Hence deterministic approach is used for inertia weight. A linearly decreasing inertia weight strategy decreases the value of inertia weight with generation number. An inertia weight value is set to 0.9 initially during explorative stage and linearly decremented to 0.4 .

\section{Population structure}

Adaptive Random topology has been defined in [30] informs $\mathrm{K}$ randomly chosen particles in swarm. . Generally $\mathrm{K}$ is set to 3 . Link modification is adaptive and depends on the fitness values at swarm level. The information links that is $\mathrm{K}$ particles are selected at the beginning, and after unsuccessful iteration. Variation of this adaptive random link is tested here. Instead of changing informants after unsuccessful iteration, informants are changed after some threshold number of unsuccessful iterations.

\section{BENCHMARK FUNCTIONS}

Both unimodal as well as multimodal benchmark functions [9] are chosen for experimentation and are listed below-

\section{- The Sphere Function}


The Sphere function is Continuous, Differentiable, Separable, Scalable and Multimodal, defined as follows-

$$
f_{1}=\sum_{i=1}^{D} x_{i}^{2}
$$

\section{- Rastrigin Function}

Rastrigin is highly multimodal and the location of the minima are regularly distributed. It is defined as -

$$
f_{2}=\sum_{i=1}^{D}\left[x_{i}^{2}-10 \cos \left(2 \pi x_{i}\right)+10\right]
$$

- Step Function

Step function is Discontinuous, Non-Differentiable, Separable, Scalable and Unimodal, , defined as follows -

$$
f_{3}=\sum_{i=1}^{D}\left(\left\lfloor x_{i}+0.5\right\rfloor\right)^{2}
$$

- Rosenbrock's Function

Rosenbrock's function is Continuous, Differentiable, Non-Separable, Scalable and Unimodal, defined as follows:

$$
f_{4}=\sum_{i=1}^{D}\left[100\left(x_{i}^{2}-x_{i+1}\right)^{2}+\left(1-x_{i}\right)^{2}\right]
$$

Where $\mathrm{D}$ is the dimension and $x=\left(x_{1}, x_{2}, \cdots, x_{\mathrm{D}}\right)$ is $\mathrm{D}$-dimensional row vector.

\section{EXPERIMENTAL SETUP}

The local best PSO is used for experimental tests and parameters are set as discussed below -

\section{- Initial population}

The positions for a particle in the swarm are initialized using uniform distribution along each dimension of the problem space.

\section{- Swarm Size}

Swarm size is considered as 40 .

- Inertia Weight

An inertia weight value starting from 0.9 linearly decreasing to 0.4 improves the performance [12], [14].

- Velocity

Initially velocity is set to zero

- Acceleration coefficient $\mathrm{c} 1$ and $\mathrm{c} 2$ are set to 1.4 .

\section{- Neighborhood Topologies}

In PSO, each particle has communication neighborhood. Following neighborhood topologies are used -

○ Adaptive Random Link

○ Adaptive Random Link with link change after threshold number of unsuccessful iterations.

\section{- Bound Handling}

As discussed by Clerc Hyperbolic and random back give better results. Hence the work considers these two prevention methods and all repositioning methods are verified with these two prevention methods for adaptive random link.

Velocity Bound Handling

1: Random back

\section{2: Hyperbolic \\ Position Bound Handling \\ 1: Nearest \\ 2: Do not change position \\ 3: Reflex}

The variants used for experiments are -

1. Adaptive random link(RL) with link change after unsuccessful iterations

Adaptive random link(RL) with links change after 10 , $20,30, \ldots, 100$ unsuccessful iterations $1 c 10,1 \mathrm{l} 20, \ldots$, 1c100 represents Link Change after 10 unsuccessful

\begin{tabular}{|c|c|c|c|}
\hline & $\begin{array}{l}\text { Neighbourhood } \\
\text { Topologies }\end{array}$ & $\begin{array}{c}\text { Velocity } \\
\text { confinement }\end{array}$ & $\begin{array}{c}\text { Position } \\
\text { confinement }\end{array}$ \\
\hline RL11 & \multirow{6}{*}{$\begin{array}{l}\text { Adaptive } \\
\text { random link }\end{array}$} & \multirow{3}{*}{ Random back } & Nearest \\
\hline RL12 & & & Do not change \\
\hline RL13 & & & Reflex \\
\hline RL21 & & \multirow{3}{*}{ Hyperbolic } & Nearest \\
\hline RL22 & & & Do not change \\
\hline RL23 & & & Reflex \\
\hline
\end{tabular}
iterations, 20 unsuccessful iterations,..., 100 unsuccessful iterations respectively.

Table 1. The variants used for experiments

The procedure is iterative. Stopping criteria is maximum number of iterations (5000) or acceptable error between optimal solution known and calculated as $10 \mathrm{E}$ 20.

\section{VIII.RESULTS AND DISCUSSION}

The experiments are carried out on both unimodal and multimodal functions. Ten runs are taken for each. Average number of iterations required to obtain acceptable solution are summarized in table 2, 3, 4, 5, 6 and 7 . Bold faced values represent minimum number of iterations required for link change after threshold number of unsuccessful iterations (Row minimum).

Table 2. Average number of iterations for random link Threshold (threshold ranging from 10 to 100) for two dimensional Sphere function

\begin{tabular}{|c|c|c|c|c|c|c|}
\hline $\begin{array}{c}\text { Link } \\
\text { change } \\
\text { threshold }\end{array}$ & RL11 & RL12 & RL13 & RL21 & RL22 & RL23 \\
\hline 1 & 573 & $\mathbf{5 3 6}$ & 547 & 575 & 537 & 571 \\
\hline 10 & 564 & 527 & 535 & 571 & 549 & $\mathbf{5 2 4}$ \\
\hline 20 & 599 & 552 & 557 & 547 & $\mathbf{5 3 4}$ & 541 \\
\hline 30 & $\mathbf{5 5 7}$ & 580 & 603 & 592 & 563 & 593 \\
\hline 40 & 553 & 560 & $\mathbf{5 4 9}$ & 578 & 576 & 559 \\
\hline 50 & 611 & 574 & 581 & $\mathbf{5 5 2}$ & 624 & 564 \\
\hline 60 & $\mathbf{5 2 9}$ & 596 & 562 & 563 & 560 & 573 \\
\hline 70 & 566 & 626 & $\mathbf{5 3 4}$ & 579 & 555 & 535 \\
\hline 80 & $\mathbf{5 5 9}$ & 594 & 592 & 608 & 565 & 563 \\
\hline 90 & 607 & 582 & 599 & 611 & 585 & $\mathbf{5 8 0}$ \\
\hline 100 & 594 & 593 & 574 & 574 & 616 & $\mathbf{5 5 3}$ \\
\hline
\end{tabular}


Table 3. Average Number Of Iterations For Random Link Threshold (Threshold Ranging From 10 To 100) For Two Dimensional Rastrigin Function

\begin{tabular}{|c|c|c|c|c|c|c|}
\hline $\begin{array}{c}\text { Link } \\
\text { change } \\
\text { threshold }\end{array}$ & RL11 & RL12 & RL13 & RL21 & RL22 & RL23 \\
\hline 1 & $\mathbf{1 8 4 6}$ & 1957 & 1959 & 2021 & 2087 & 2046 \\
\hline 10 & 1970 & 1815 & 1800 & 1761 & 1867 & $\mathbf{1 7 4 6}$ \\
\hline 20 & 1944 & 1946 & 1988 & 1760 & 1804 & $\mathbf{1 6 6 9}$ \\
\hline 30 & 2013 & 1627 & 1888 & $\mathbf{1 5 4 2}$ & 1852 & 1827 \\
\hline 40 & $\mathbf{9 7 9}$ & 1418 & 1126 & 1133 & 1092 & 1209 \\
\hline 50 & 1001 & 1301 & $\mathbf{9 2 1}$ & 1137 & 994 & 967 \\
\hline 60 & 1055 & 1081 & $\mathbf{8 5 7}$ & 1103 & 943 & 954 \\
\hline 70 & 1019 & 1020 & $\mathbf{9 9 3}$ & 1021 & 1007 & 1100 \\
\hline 80 & 1056 & 1034 & 1081 & $\mathbf{8 1 5}$ & 877 & 985 \\
\hline 90 & 938 & 1059 & 1069 & 969 & 1027 & $\mathbf{9 3 5}$ \\
\hline 100 & 1066 & 962 & $\mathbf{8 6 9}$ & 1134 & 1045 & 1049 \\
\hline
\end{tabular}

Table 4. Average number of iterations for random link Threshold (threshold ranging from 10 to 100) for two dimensional Step function

\begin{tabular}{|c|c|c|c|c|c|c|}
\hline $\begin{array}{c}\text { Link } \\
\text { change } \\
\text { threshold }\end{array}$ & RL11 & RL12 & RL13 & RL21 & RL22 & RL23 \\
\hline 1 & $\mathbf{3 7}$ & 38 & 41 & 48 & 44 & 46 \\
\hline 10 & 47 & 46 & 44 & $\mathbf{3 7}$ & 49 & 40 \\
\hline 20 & 54 & $\mathbf{3 8}$ & 44 & 48 & 46 & 46 \\
\hline 30 & 51 & 47 & $\mathbf{3 5}$ & 47 & 46 & 57 \\
\hline 40 & 47 & 37 & 45 & 53 & 47 & $\mathbf{3 6}$ \\
\hline 50 & 43 & 50 & 42 & 42 & $\mathbf{3 8}$ & 45 \\
\hline 60 & 43 & 40 & 45 & 44 & 49 & $\mathbf{3 7}$ \\
\hline 70 & 56 & 52 & 49 & 42 & $\mathbf{4 1}$ & 47 \\
\hline 80 & 49 & 47 & 45 & 48 & 45 & $\mathbf{3 8}$ \\
\hline 90 & 45 & 47 & 53 & 56 & 45 & $\mathbf{4 3}$ \\
\hline 100 & 48 & 43 & $\mathbf{3 1}$ & $\mathbf{4 3}$ & 43 & 37 \\
\hline
\end{tabular}

Table 5. Average number of iterations for random link Threshold (threshold ranging from 10 to 100) for two dimensional Rosenbrock function

\begin{tabular}{|c|c|c|c|c|c|c|}
\hline $\begin{array}{c}\text { Link } \\
\text { change } \\
\text { threshold }\end{array}$ & RL11 & RL12 & RL13 & RL21 & RL22 & RL23 \\
\hline 1 & 3471 & 3427 & 3418 & $\mathbf{3 2 3 9}$ & 3311 & 3480 \\
\hline 10 & 3540 & 3589 & 3447 & $\mathbf{3 3 7 1}$ & 3457 & 3372 \\
\hline 20 & 3425 & $\mathbf{3 3 2 7}$ & 3416 & 3425 & 3477 & 3484 \\
\hline 30 & 3181 & 3401 & $\mathbf{3 0 1 5}$ & 3159 & 3097 & 3191 \\
\hline 40 & 2509 & $\mathbf{2 4 8 5}$ & 2843 & 2859 & 2870 & 3040 \\
\hline 50 & 2504 & 2077 & 2179 & $\mathbf{2 0 2 9}$ & 2372 & 2153 \\
\hline 60 & $\mathbf{1 9 4 4}$ & 2207 & 2075 & 1948 & 2077 & 2089 \\
\hline 70 & 2087 & $\mathbf{2 0 8 1}$ & 2136 & 2138 & 2166 & 2131 \\
\hline 80 & 2206 & 2065 & 2043 & 2087 & 2040 & $\mathbf{2 0 3 7}$ \\
\hline 90 & 2127 & 2191 & $\mathbf{2 0 3 1}$ & 2224 & 2112 & 2151 \\
\hline 100 & 2196 & $\mathbf{2 0 6 9}$ & 2182 & 2271 & 2213 & 2182 \\
\hline
\end{tabular}

The variations in the average number of iterations required for adaptive random link variants with threshold ranging from 1 to 100 is shown in fig. 1 .

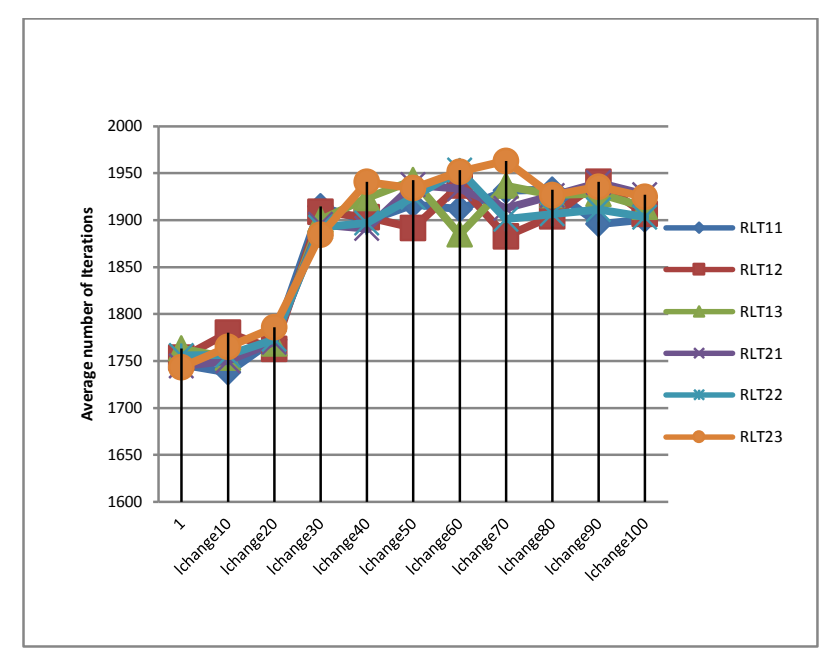

Fig.1. Average number of iterations for random link Threshold (threshold ranging from 10 to 100) for ten dimensional Sphere function

Table 6. Average number of iterations for random link Threshold (threshold ranging from 10 to 100) for ten dimensional Sphere function

\begin{tabular}{|c|c|c|c|c|c|c|}
\hline $\begin{array}{c}\text { Link } \\
\text { change } \\
\text { threshold }\end{array}$ & RL11 & RL12 & RL13 & RL21 & RL22 & RL23 \\
\hline lc1 & 1746 & 1753 & 1763 & 1745 & 1756 & $\mathbf{1 7 4 4}$ \\
\hline lc10 & $\mathbf{1 7 3 8}$ & 1780 & 1754 & 1750 & 1756 & 1765 \\
\hline lc20 & 1773 & $\mathbf{1 7 6 3}$ & 1769 & 1770 & 1773 & 1786 \\
\hline lc30 & 1915 & 1909 & 1900 & 1896 & 1892 & $\mathbf{1 8 8 4}$ \\
\hline lc40 & 1899 & 1903 & 1924 & $\mathbf{1 8 9 1}$ & 1897 & 1941 \\
\hline lc50 & 1917 & $\mathbf{1 8 9 2}$ & 1942 & 1938 & 1925 & 1934 \\
\hline lc60 & 1912 & 1937 & $\mathbf{1 8 8 5}$ & 1933 & 1953 & 1952 \\
\hline lc70 & 1931 & $\mathbf{1 8 8 3}$ & 1937 & 1913 & 1901 & 1963 \\
\hline lc80 & 1932 & $\mathbf{1 9 0 4}$ & 1927 & 1926 & 1907 & 1927 \\
\hline lc90 & $\mathbf{1 8 9 6}$ & 1941 & 1928 & 1940 & 1911 & 1936 \\
\hline lc100 & $\mathbf{1 9 0 1}$ & 1907 & 1914 & 1927 & 1903 & 1925 \\
\hline
\end{tabular}

Table 7. Average number of iterations for random link Threshold (threshold ranging from 10 to 100) for ten dimensional Step function

\begin{tabular}{|c|c|c|c|c|c|c|}
\hline $\begin{array}{c}\text { Link } \\
\text { Change } \\
\text { threshold }\end{array}$ & RL11 & RL12 & RL13 & RL21 & RL22 & RL23 \\
\hline lc1 & 772 & 768 & $\mathbf{7 2 1}$ & 798 & 778 & 786 \\
\hline lc10 & 792 & 758 & 789 & 828 & $\mathbf{7 4 7}$ & 804 \\
\hline lc20 & $\mathbf{7 2 9}$ & 783 & 794 & 759 & 762 & 779 \\
\hline lc30 & 864 & $\mathbf{7 9 4}$ & 849 & 822 & 841 & 808 \\
\hline lc40 & $\mathbf{8 2 3}$ & 865 & 847 & 859 & 837 & 850 \\
\hline lc50 & 944 & 900 & 946 & 937 & 891 & $\mathbf{8 7 3}$ \\
\hline lc60 & $\mathbf{8 5 1}$ & 932 & 864 & 877 & 862 & 926 \\
\hline lc70 & 896 & 869 & $\mathbf{8 6 7}$ & 899 & 929 & 941 \\
\hline lc80 & 907 & 929 & 909 & 939 & 943 & $\mathbf{8 9 0}$ \\
\hline lc90 & 957 & 943 & 988 & 908 & 901 & $\mathbf{8 7 3}$ \\
\hline lc100 & 915 & 939 & 916 & 917 & 955 & $\mathbf{9 0 2}$ \\
\hline
\end{tabular}

Table 8 and 9 focuses on minimum value obtained for benchmark function. Standard deviation shows variation for type of functions and link change threshold. Tables 10, 11,12 and 13 Statistical evaluation.

I.J. Intelligent Systems and Applications, 2015, 01, 62-72 
Table 8. Min Value Obtained (Threshold Ranging From 1 To 100) For Ten Dimensional Sphere Function

\begin{tabular}{|c|c|c|c|c|c|c|}
\hline Link change threshold & RLT11 & RLT12 & RLT13 & RLT21 & RLT22 & RLT23 \\
\hline 1 & $8.84 \mathrm{E}-21$ & $7.9 \mathrm{E}-21$ & $6 \mathrm{E}-21$ & $8.41 \mathrm{E}-21$ & $2.1 \mathrm{E}-21$ & $6.6 \mathrm{E}-21$ \\
\hline 10 & $8.24 \mathrm{E}-21$ & $6.1 \mathrm{E}-21$ & $6.3 \mathrm{E}-21$ & $4.96 \mathrm{E}-21$ & $5.9 \mathrm{E}-21$ & $4.6 \mathrm{E}-21$ \\
\hline 20 & $5.11 \mathrm{E}-21$ & $6.5 \mathrm{E}-21$ & $6.3 \mathrm{E}-21$ & $7.06 \mathrm{E}-21$ & $6.6 \mathrm{E}-21$ & $7.1 \mathrm{E}-21$ \\
\hline 30 & $6.54 \mathrm{E}-21$ & $4.5 \mathrm{E}-21$ & $5.9 \mathrm{E}-21$ & $6.85 \mathrm{E}-21$ & $4.7 \mathrm{E}-21$ & $7.6 \mathrm{E}-21$ \\
\hline 40 & $7.6 \mathrm{E}-21$ & $8.3 \mathrm{E}-21$ & $7.2 \mathrm{E}-21$ & $5.47 \mathrm{E}-21$ & $5.5 \mathrm{E}-21$ & $7.1 \mathrm{E}-21$ \\
\hline 50 & $5.11 \mathrm{E}-21$ & $7.1 \mathrm{E}-21$ & $7.9 \mathrm{E}-21$ & $5.98 \mathrm{E}-21$ & $5.6 \mathrm{E}-21$ & $7.1 \mathrm{E}-21$ \\
\hline 60 & $5.95 \mathrm{E}-21$ & $2.7 \mathrm{E}-21$ & $4.9 \mathrm{E}-21$ & $5.54 \mathrm{E}-21$ & $4.4 \mathrm{E}-21$ & $5.7 \mathrm{E}-21$ \\
\hline 70 & $5.21 \mathrm{E}-21$ & $8.1 \mathrm{E}-21$ & $7.3 \mathrm{E}-21$ & $4.9 \mathrm{E}-21$ & $7.3 \mathrm{E}-21$ & $6.5 \mathrm{E}-21$ \\
\hline 80 & $4.36 \mathrm{E}-21$ & $5.7 \mathrm{E}-21$ & $5.3 \mathrm{E}-21$ & $6.21 \mathrm{E}-21$ & $5.8 \mathrm{E}-21$ & $8.5 \mathrm{E}-21$ \\
\hline 90 & $3.85 \mathrm{E}-21$ & $4.7 \mathrm{E}-21$ & $7.1 \mathrm{E}-21$ & $4.19 \mathrm{E}-21$ & $3.1 \mathrm{E}-21$ & $7.5 \mathrm{E}-21$ \\
\hline 100 & $7.59 \mathrm{E}-21$ & $7.5 \mathrm{E}-21$ & $4.8 \mathrm{E}-21$ & $7.67 \mathrm{E}-21$ & $6.2 \mathrm{E}-21$ & $7.8 \mathrm{E}-21$ \\
\hline
\end{tabular}

Table 9. Min Value Obtained (Threshold Ranging From 1 To 100) For Ten Dimensional Step Function

\begin{tabular}{|c|c|c|c|c|c|c|}
\hline $\begin{array}{c}\text { Link change } \\
\text { threshold }\end{array}$ & $\begin{array}{c}\text { RLT } \\
11\end{array}$ & $\begin{array}{c}\text { RLT } \\
12\end{array}$ & $\begin{array}{c}\text { RLT } \\
13\end{array}$ & $\begin{array}{c}\text { RLT } \\
21\end{array}$ & $\begin{array}{c}\text { RLT } \\
22\end{array}$ & $\begin{array}{c}\text { RLT } \\
23\end{array}$ \\
\hline 1 & 0 & 0 & 0 & 0 & 0 & 0 \\
\hline 10 & 0 & 0 & 0 & 0 & 0 & 0 \\
\hline 20 & 0 & 0 & 0 & 0 & 0 & 0 \\
\hline 30 & 0 & 0 & 0 & 0 & 0 & 0 \\
\hline 40 & 0 & 0 & 0 & 0 & 0 & 0 \\
\hline 50 & 0 & 0 & 0 & 0 & 0 & 0 \\
\hline 60 & 0 & 0 & 0 & 0 & 0 & 0 \\
\hline 70 & 0 & 0 & 0 & 0 & 0 & 0 \\
\hline 80 & 0 & 0 & 0 & 0 & 0 & 0 \\
\hline 90 & 0 & 0 & 0 & 0 & 0 & 0 \\
\hline 100 & 0 & 0 & 0 & 0 & 0 & 0 \\
\hline
\end{tabular}

Table 10. Statistical Evaluation for Two Dimensional Sphere Function Based On Average Number for Iterations

\begin{tabular}{|c|c|c|c|c|c|}
\hline $\begin{array}{c}\text { Link change } \\
\text { threshold }\end{array}$ & Min. & Median & Mean & Max. & $\begin{array}{c}\text { Std. } \\
\text { Deviation }\end{array}$ \\
\hline 1 & 536 & 559 & 557 & 575 & 19 \\
\hline 10 & 524 & 542 & 545 & 571 & 20 \\
\hline 20 & 534 & 550 & 555 & 599 & 23 \\
\hline 30 & 557 & 586 & 581 & 603 & 18 \\
\hline 40 & 549 & 560 & 563 & 578 & 12 \\
\hline 50 & 552 & 578 & 584 & 624 & 28 \\
\hline 60 & 529 & 563 & 564 & 596 & 22 \\
\hline 70 & 534 & 561 & 566 & 626 & 34 \\
\hline 80 & 559 & 579 & 580 & 608 & 20 \\
\hline 90 & 580 & 592 & 594 & 611 & 13 \\
\hline 100 & 553 & 584 & 584 & 616 & 22 \\
\hline
\end{tabular}

Interpretation of correlation coefficient -

Correlation analysis is used to study the interdependence of two variables. If large (small) values are associated with large (small) values of other variable then there exists strong positive correlation. If large (small) values are associated with small (large) values of other variable then there exists strong negative correlation. 1. If correlation coefficient is 1 then perfect positive linear relationship exists between two variables.

Copyright (C) 2015 MECS
2. If correlation coefficient is -1 then perfect negative linear relationship exists between two variables

3. If correlation coefficient is greater than or equal to 0.7 and less than or equal to 1 then strong positive linear relationship exists between two variables

4. If correlation coefficient is greater than or equal to -1 and less than or equal to -0.7 then strong negative linear relationship exists between two variables

5. If correlation coefficient is 0 then no linear relationship between two variables exists between two variables

Table 11. Statistical evaluation for two dimensional Rastrgin function based on average number for iterations

\begin{tabular}{|c|c|c|c|c|c|}
\hline $\begin{array}{c}\text { Link change } \\
\text { threshold }\end{array}$ & Min. & Median & Mean & Max. & $\begin{array}{c}\text { std } \\
\text { dev }\end{array}$ \\
\hline 1 & 536 & 559 & 557 & 575 & 85 \\
\hline 10 & 524 & 542 & 545 & 571 & 82 \\
\hline 20 & 534 & 550 & 555 & 599 & 127 \\
\hline 30 & 557 & 586 & 581 & 603 & 175 \\
\hline 40 & 549 & 560 & 563 & 578 & 147 \\
\hline 50 & 552 & 578 & 584 & 624 & 141 \\
\hline 60 & 529 & 563 & 564 & 596 & 96 \\
\hline 70 & 534 & 561 & 566 & 626 & 38 \\
\hline 80 & 559 & 579 & 580 & 608 & 106 \\
\hline 90 & 580 & 592 & 594 & 611 & 60 \\
\hline 100 & 553 & 584 & 584 & 616 & 92 \\
\hline
\end{tabular}

Table 12. Statistical Evaluation for Two Dimensional Step Function Based On Average Number for Iterations

\begin{tabular}{|c|c|c|c|c|c|}
\hline $\begin{array}{c}\text { Link change } \\
\text { threshold }\end{array}$ & Min. & Median & Mean & Max. & $\begin{array}{c}\text { Std. } \\
\text { Deviation }\end{array}$ \\
\hline 1 & 37 & 43 & 42 & 48 & 4 \\
\hline 10 & 37 & 45 & 44 & 49 & 5 \\
\hline 20 & 38 & 46 & 46 & 54 & 5 \\
\hline 30 & 35 & 47 & 47 & 57 & 7 \\
\hline 40 & 36 & 46 & 44 & 53 & 7 \\
\hline 50 & 38 & 43 & 43 & 50 & 4 \\
\hline 60 & 37 & 44 & 43 & 49 & 4 \\
\hline 70 & 41 & 48 & 48 & 56 & 6 \\
\hline 80 & 38 & 46 & 45 & 49 & 4 \\
\hline 90 & 43 & 46 & 48 & 56 & 5 \\
\hline 100 & 31 & 43 & 41 & 48 & 6 \\
\hline
\end{tabular}

I.J. Intelligent Systems and Applications, 2015, 01, 62-72 
Table 13. Statistical evaluation for two dimensional Rosenbrock function based on average number for iterations

\begin{tabular}{|c|c|c|c|c|c|}
\hline $\begin{array}{c}\text { Link change } \\
\text { threshold }\end{array}$ & Min. & Median & Mean & Max. & $\begin{array}{c}\text { std } \\
\text { dev. }\end{array}$ \\
\hline 1 & 3239 & 3422 & 3391 & 3480 & 96 \\
\hline 10 & 3371 & 3452 & 3463 & 3589 & 88 \\
\hline 20 & 3327 & 3425 & 3426 & 3484 & 56 \\
\hline 30 & 3015 & 3170 & 3174 & 3401 & 129 \\
\hline 40 & 2485 & 2851 & 2768 & 3040 & 222 \\
\hline 50 & 2029 & 2166 & 2219 & 2504 & 183 \\
\hline 60 & 1944 & 2076 & 2057 & 2207 & 99 \\
\hline 70 & 2081 & 2134 & 2123 & 2166 & 33 \\
\hline 80 & 2037 & 2054 & 2080 & 2206 & 65 \\
\hline 90 & 2031 & 2139 & 2139 & 2224 & 67 \\
\hline 100 & 2069 & 2189 & 2186 & 2271 & 66 \\
\hline & & & & & \\
\hline
\end{tabular}

Table 14. Correlation For Two Dimensional Sphere Function With Link Change From 1 To 100.

\begin{tabular}{|c|c|c|c|c|c|c|}
\hline $\begin{array}{c}\text { Link } \\
\text { change } \\
\text { threshold }\end{array}$ & RL11 & RL12 & RL13 & RL21 & RL22 & RL23 \\
\hline 1 & $\mathbf{1 . 0 0}$ & $\mathbf{1 . 0 0}$ & $\mathbf{1 . 0 0}$ & $\mathbf{1 . 0 0}$ & $\mathbf{1 . 0 0}$ & $\mathbf{1 . 0 0}$ \\
\hline 10 & $\mathbf{0 . 9 9}$ & $\mathbf{0 . 8 6}$ & $\mathbf{0 . 9 8}$ & $\mathbf{0 . 8 9}$ & $\mathbf{0 . 9 6}$ & $\mathbf{0 . 8 8}$ \\
\hline 20 & -0.10 & $\mathbf{0 . 7 6}$ & 0.61 & -0.03 & $\mathbf{0 . 8 9}$ & 0.41 \\
\hline 30 & 0.59 & 0.27 & 0.02 & 0.31 & -0.44 & -0.24 \\
\hline 40 & 0.28 & $\mathbf{0 . 8 9}$ & -0.13 & 0.71 & 0.44 & 0.32 \\
\hline 50 & 0.25 & 0.16 & 0.12 & 0.68 & 0.38 & $\mathbf{0 . 8 2}$ \\
\hline 60 & 0.61 & -0.22 & -0.18 & -0.37 & 0.52 & 0.51 \\
\hline 70 & 0.15 & 0.25 & 0.06 & 0.60 & -0.01 & -0.23 \\
\hline 80 & 0.26 & 0.26 & -0.58 & NA & NA & 0.05 \\
\hline 90 & 0.13 & 0.01 & -0.15 & NA & 0.55 & 0.63 \\
\hline 100 & NA & 0.22 & NA & -0.05 & NA & 0.15 \\
\hline & & & & & & \\
\hline
\end{tabular}

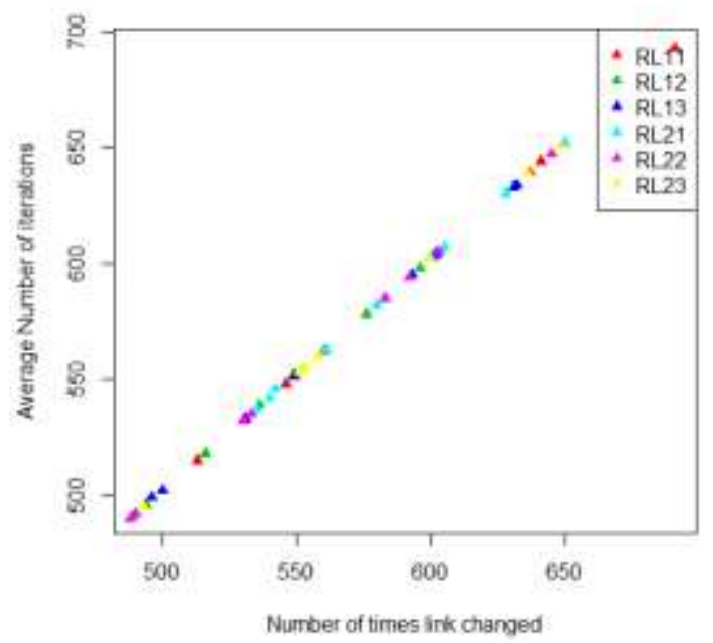

Fig. 2. Average number of iterations vs Number of times link changed for sphere Function D2 link change after every unsuccessful iteration

Figures 2 to 12 show plots of average number of iterations against number of times link changed for benchmark functions depending on iterations.

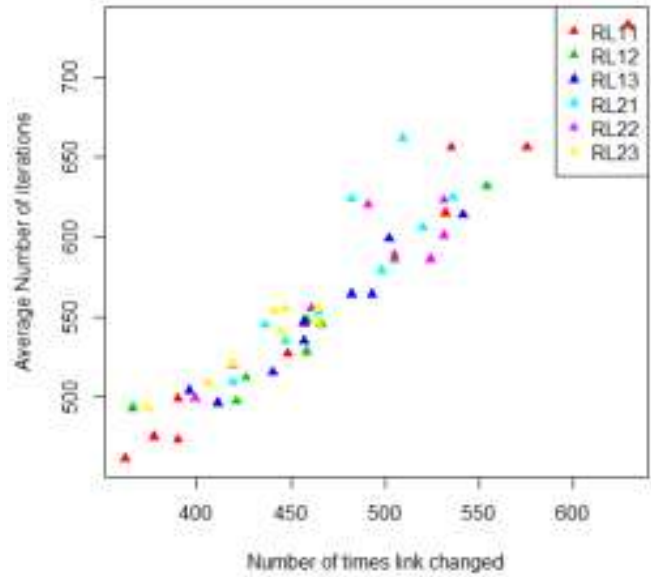

Fig.3. Average number of iterations vs Number of times link changed for sphere Function D2 link change after 10 unsuccessful iteration

Table 15. Correlation For Two Dimensional Rastrigin Function With Link Change From 1 To 100.

\begin{tabular}{|c|c|c|c|c|c|c|}
\hline $\begin{array}{c}\text { Link } \\
\text { change } \\
\text { threshold }\end{array}$ & RL11 & RL12 & RL13 & RL21 & RL22 & RL23 \\
\hline 1 & $\mathbf{1 . 0 0}$ & $\mathbf{1 . 0 0}$ & $\mathbf{1 . 0 0}$ & $\mathbf{1 . 0 0}$ & $\mathbf{1 . 0 0}$ & $\mathbf{1 . 0 0}$ \\
\hline 10 & $\mathbf{1 . 0 0}$ & $\mathbf{1 . 0 0}$ & $\mathbf{1 . 0 0}$ & $\mathbf{1 . 0 0}$ & $\mathbf{1 . 0 0}$ & $\mathbf{1 . 0 0}$ \\
\hline 20 & $\mathbf{1 . 0 0}$ & $\mathbf{0 . 9 8}$ & $\mathbf{0 . 9 9}$ & $\mathbf{1 . 0 0}$ & $\mathbf{1 . 0 0}$ & $\mathbf{0 . 9 9}$ \\
\hline 30 & $\mathbf{0 . 9 9}$ & $\mathbf{1 . 0 0}$ & $\mathbf{1 . 0 0}$ & $\mathbf{0 . 9 9}$ & $\mathbf{0 . 9 9}$ & $\mathbf{0 . 9 9}$ \\
\hline 40 & $\mathbf{0 . 8 3}$ & $\mathbf{0 . 9 9}$ & $\mathbf{0 . 9 6}$ & $\mathbf{0 . 8 6}$ & $\mathbf{0 . 9 0}$ & $\mathbf{0 . 9 2}$ \\
\hline 50 & $\mathbf{0 . 9 8}$ & $\mathbf{0 . 9 2}$ & $\mathbf{0 . 9 1}$ & $\mathbf{0 . 8 9}$ & $\mathbf{0 . 8 6}$ & $\mathbf{0 . 9 2}$ \\
\hline 60 & $\mathbf{0 . 8 4}$ & $\mathbf{0 . 9 3}$ & $\mathbf{0 . 7 4}$ & $\mathbf{0 . 9 4}$ & $\mathbf{0 . 8 3}$ & $\mathbf{0 . 8 4}$ \\
\hline 70 & $\mathbf{0 . 8 5}$ & $\mathbf{0 . 8 6}$ & $\mathbf{0 . 9 3}$ & $\mathbf{0 . 8 8}$ & $\mathbf{0 . 6 2}$ & $\mathbf{0 . 7 4}$ \\
\hline 80 & $\mathbf{0 . 8 1}$ & $\mathbf{0 . 8 5}$ & 0.08 & $\mathbf{0 . 8 2}$ & 0.63 & $\mathbf{0 . 9 5}$ \\
\hline 90 & $\mathbf{0 . 8 9}$ & 0.63 & 0.60 & $\mathbf{0 . 9 2}$ & $\mathbf{0 . 9 1}$ & $\mathbf{0 . 8 0}$ \\
\hline 100 & $\mathbf{0 . 8 2}$ & $\mathbf{0 . 7 6}$ & $\mathbf{0 . 9 0}$ & $\mathbf{0 . 7 7}$ & $\mathbf{0 . 8 8}$ & 0.34 \\
\hline
\end{tabular}

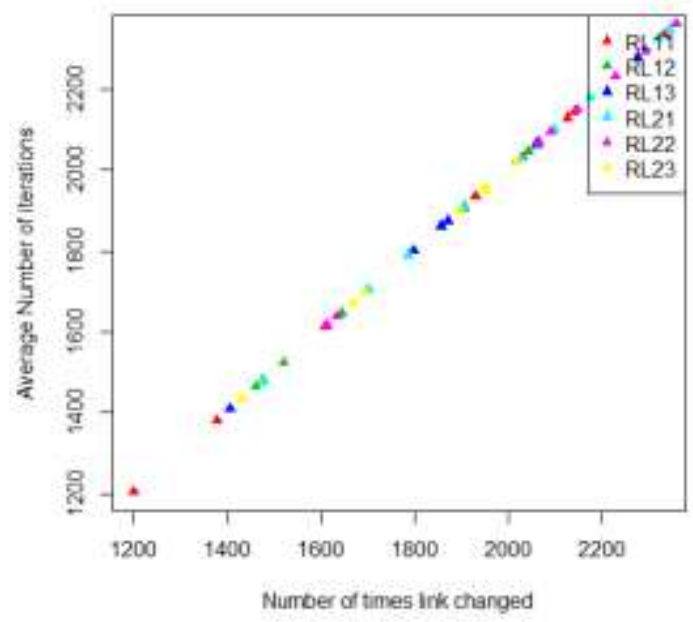

Fig. 4. Average number of iterations vs Number of times link changed for Rastrigin Function D2 link change after every unsuccessful iteration

I.J. Intelligent Systems and Applications, 2015, 01, 62-72 


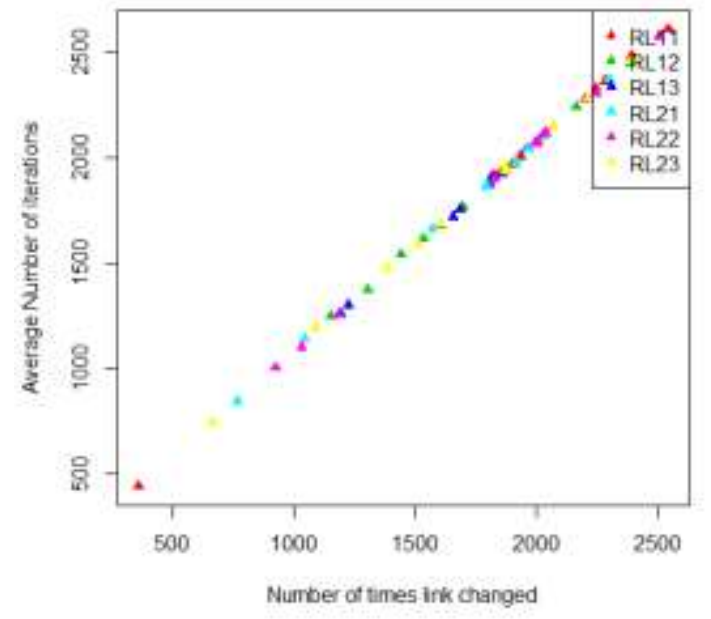

Fig. 5. Average number of iterations vs Number of times link changed for two dimensional Rastrigin Function link change after 10 unsuccessful iteration

Table 16. Correlation for Two Dimensional Step Function

\begin{tabular}{|c|c|c|c|c|c|c|}
\hline $\begin{array}{c}\text { Link } \\
\text { change } \\
\text { threshold }\end{array}$ & RL11 & RL12 & RL13 & RL21 & RL22 & RL23 \\
\hline 1 & $\mathbf{1 . 0 0}$ & $\mathbf{1 . 0 0}$ & $\mathbf{1 . 0 0}$ & $\mathbf{1 . 0 0}$ & $\mathbf{1 . 0 0}$ & $\mathbf{1 . 0 0}$ \\
\hline 10 & $\mathbf{0 . 8 9}$ & $\mathbf{0 . 7 9}$ & $\mathbf{0 . 8 8}$ & $\mathbf{0 . 9 3}$ & $\mathbf{0 . 9 7}$ & $\mathbf{0 . 9 8}$ \\
\hline 20 & 0.66 & 0.48 & $\mathbf{0 . 9 3}$ & $\mathbf{0 . 8 5}$ & $\mathbf{0 . 9 2}$ & $\mathbf{0 . 7 5}$ \\
\hline 30 & 0.65 & $\mathbf{0 . 8 4}$ & NA & $\mathbf{0 . 9 5}$ & $\mathbf{0 . 7 1}$ & $\mathbf{0 . 7 3}$ \\
\hline 40 & NA & NA & $\mathbf{0 . 7 9}$ & 0.64 & NA & $\mathbf{0 . 8 3}$ \\
\hline 50 & NA & $\mathbf{0 . 7 1}$ & NA & NA & NA & NA \\
\hline 60 & NA & NA & NA & NA & NA & NA \\
\hline 70 & NA & NA & NA & NA & NA & NA \\
\hline 80 & NA & NA & NA & NA & NA & NA \\
\hline 90 & NA & NA & NA & NA & NA & NA \\
\hline 100 & NA & NA & NA & NA & NA & NA \\
\hline
\end{tabular}

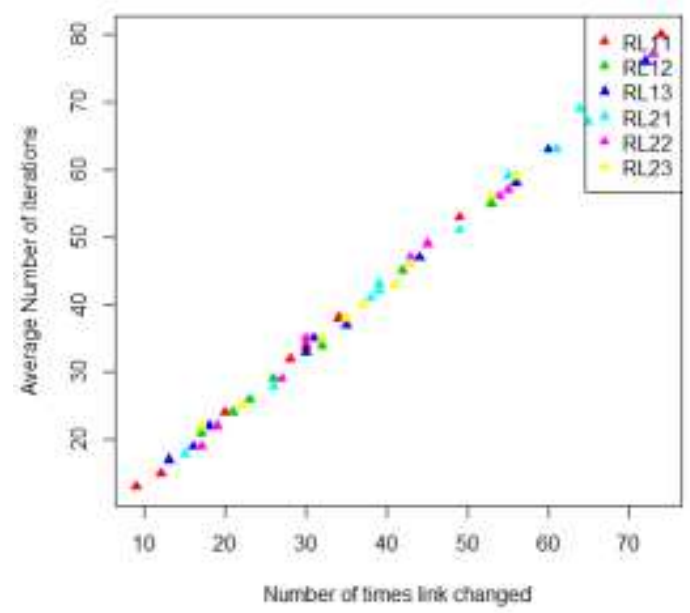

Fig. 6. Average number of iterations vs Number of times link changed for Step Function D2 link change after every unsuccessful iteration

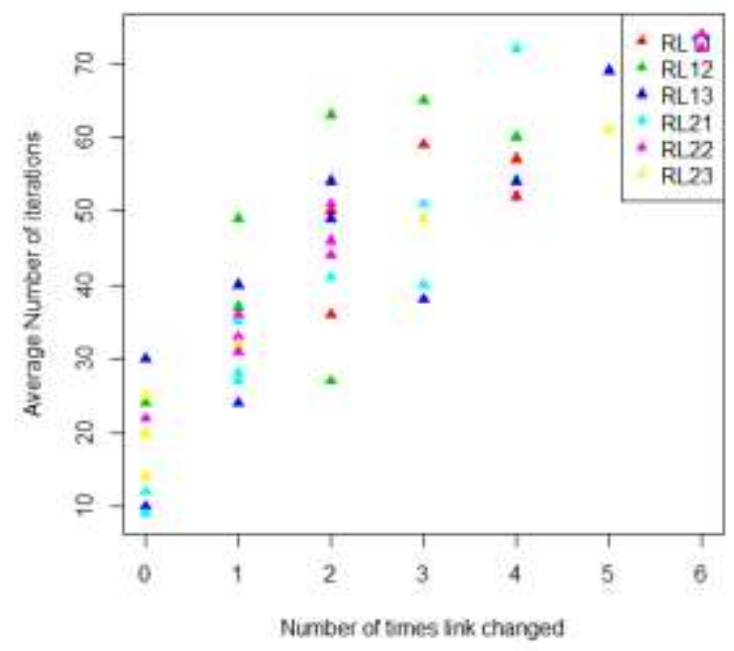

Fig.7. Average number of iterations vs Number of times link changed for Step Function D2 Link change $=10$

Table 17. Correlation for Two Dimensional Rosenbrock Function

\begin{tabular}{|c|c|c|c|c|c|c|}
\hline $\begin{array}{c}\text { Link } \\
\text { change } \\
\text { threshold }\end{array}$ & RL11 & RL12 & RL13 & RL21 & RL22 & RL23 \\
\hline 1 & $\mathbf{1 . 0 0}$ & $\mathbf{1 . 0 0}$ & $\mathbf{1 . 0 0}$ & $\mathbf{1 . 0 0}$ & $\mathbf{1 . 0 0}$ & $\mathbf{1 . 0 0}$ \\
\hline 10 & $\mathbf{0 . 9 1}$ & $\mathbf{1 . 0 0}$ & $\mathbf{1 . 0 0}$ & $\mathbf{1 . 0 0}$ & $\mathbf{1 . 0 0}$ & $\mathbf{1 . 0 0}$ \\
\hline 20 & $\mathbf{0 . 9 9}$ & $\mathbf{1 . 0 0}$ & $\mathbf{1 . 0 0}$ & $\mathbf{0 . 9 7}$ & $\mathbf{0 . 9 9}$ & $\mathbf{0 . 9 6}$ \\
\hline 30 & $\mathbf{0 . 8 6}$ & $\mathbf{0 . 9 3}$ & $\mathbf{0 . 9 8}$ & $\mathbf{0 . 9 5}$ & $\mathbf{0 . 9 9}$ & $\mathbf{0 . 9 7}$ \\
\hline 40 & $\mathbf{0 . 9 8}$ & $\mathbf{0 . 9 7}$ & $\mathbf{0 . 9 7}$ & $\mathbf{0 . 9 0}$ & $\mathbf{0 . 9 7}$ & $\mathbf{0 . 9 4}$ \\
\hline 50 & $\mathbf{0 . 9 6}$ & $\mathbf{0 . 8 0}$ & $\mathbf{0 . 9 2}$ & $\mathbf{0 . 7 6}$ & $\mathbf{0 . 9 8}$ & $\mathbf{0 . 8 9}$ \\
\hline 60 & 0.64 & $\mathbf{0 . 7 6}$ & 0.64 & 0.64 & 0.13 & $\mathbf{0 . 8 0}$ \\
\hline 70 & 0.60 & 0.57 & $\mathbf{0 . 7 4}$ & $\mathbf{0 . 7 7}$ & $\mathbf{0 . 9 8}$ & 0.48 \\
\hline 80 & $\mathbf{0 . 8 8}$ & $\mathbf{0 . 9 2}$ & 0.68 & 0.31 & 0.55 & 0.40 \\
\hline 90 & 0.62 & 0.59 & 0.59 & $\mathbf{0 . 8 9}$ & $\mathbf{0 . 8 0}$ & $\mathbf{0 . 7 3}$ \\
\hline 100 & 0.45 & 0.57 & 0.65 & 0.30 & 0.60 & $\mathbf{0 . 7 3}$ \\
\hline
\end{tabular}

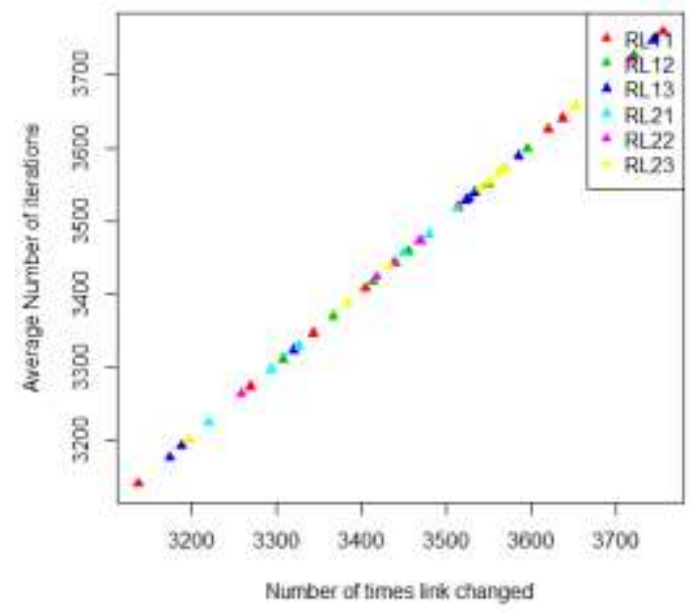

Fig. 8. Average number of iterations vs Number of times link changed for Rosenbrock Function D2 Link change $=1$ 


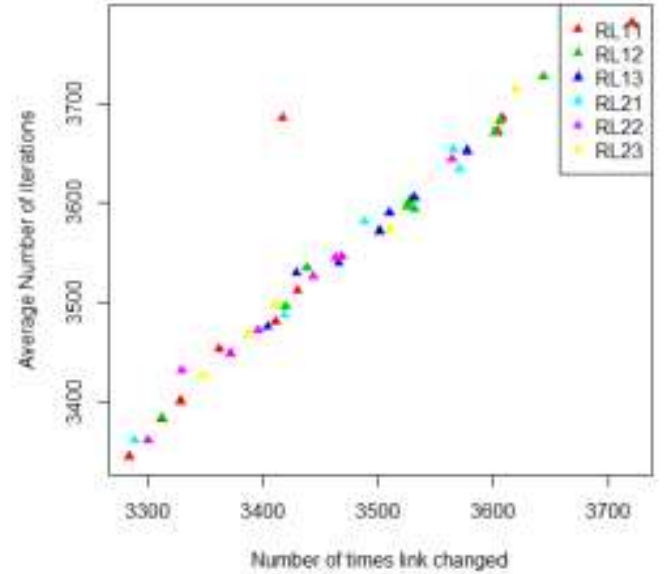

Fig.9. Average number of iterations vs Number of times link changed for Rosenbrock Function D2 with link change threshold=10

Table 18. Correlation For Ten Dimensional Sphere Function With Link Change From 20 To 100

\begin{tabular}{|c|c|c|c|c|c|c|}
\hline $\begin{array}{c}\text { Link } \\
\text { Change } \\
\text { threshold }\end{array}$ & RL11 & RL12 & RL13 & RL21 & RL22 & RL23 \\
\hline 1 & $\mathbf{1 . 0 0}$ & $\mathbf{1 . 0 0}$ & $\mathbf{1 . 0 0}$ & $\mathbf{1 . 0 0}$ & $\mathbf{1 . 0 0}$ & $\mathbf{1 . 0 0}$ \\
\hline 10 & $\mathbf{0 . 9 5}$ & $\mathbf{0 . 8 7}$ & $\mathbf{0 . 9 0}$ & $\mathbf{0 . 6 5}$ & $\mathbf{0 . 9 8}$ & $\mathbf{0 . 7 9}$ \\
\hline 20 & 0.47 & 0.29 & 0.41 & 0.32 & 0.30 & 0.47 \\
\hline 30 & 0.09 & -0.21 & 0.18 & 0.20 & $\mathbf{0 . 7 8}$ & -0.31 \\
\hline 40 & 0.07 & 0.37 & -0.02 & -0.45 & 0.09 & -0.44 \\
\hline 50 & 0.16 & -0.55 & 0.06 & -0.27 & 0.27 & -0.47 \\
\hline 60 & 0.22 & -0.15 & -0.28 & -0.15 & 0.56 & -0.55 \\
\hline 70 & $\mathbf{0 . 7 9}$ & 0.48 & 0.17 & -0.08 & 0.32 & 0.69 \\
\hline 80 & 0.25 & 0.27 & 0.55 & 0.59 & 0.02 & 0.16 \\
\hline 90 & 0.35 & $\mathbf{0 . 8 1}$ & 0.01 & -0.49 & -0.44 & -0.06 \\
\hline 100 & 0.51 & $\mathbf{0 . 7 3}$ & -0.63 & -0.32 & 0.49 & NA \\
\hline
\end{tabular}

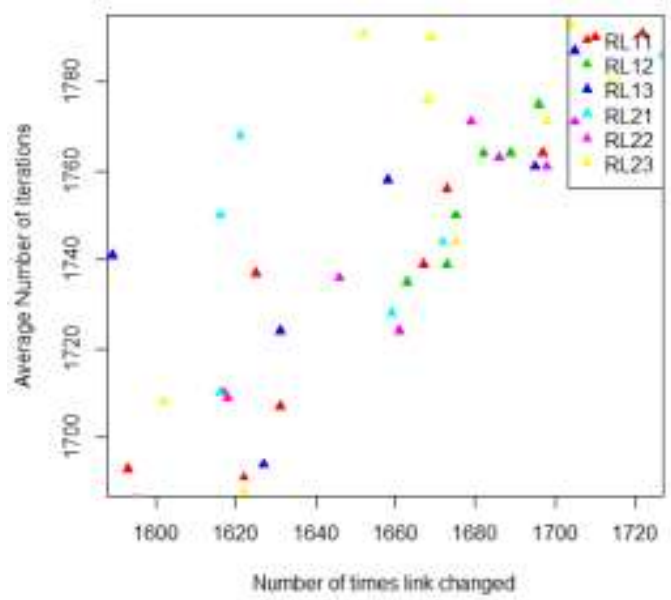

Fig. 10 Average number of iterations vs Number of times link changed for Sphere Function D10 with link change threshold=10

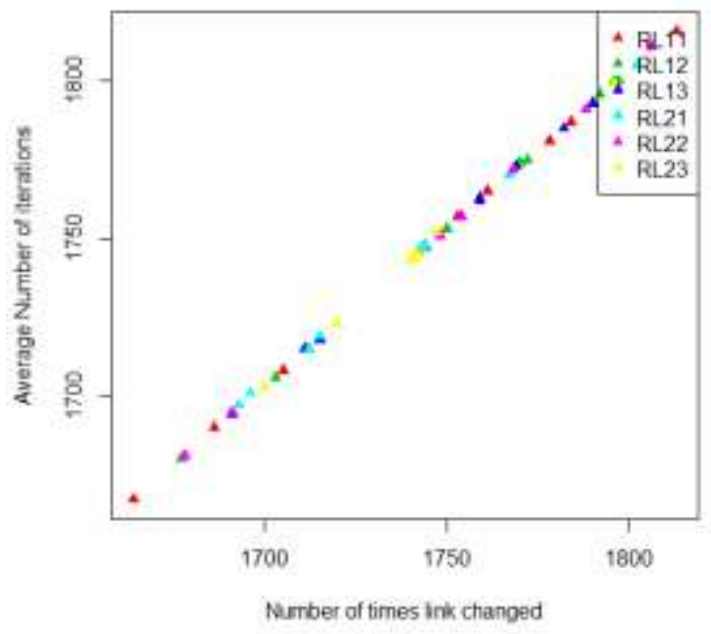

Fig. 11. Average number of iterations vs Number of times link changed for Sphere Function D10 with link change threshold=1

Table 19. Correlation For Ten Dimensional Step Function With Link Change From1 To 100

\begin{tabular}{|c|c|c|c|c|c|c|}
\hline $\begin{array}{c}\text { Link } \\
\text { change } \\
\text { threshold }\end{array}$ & RL11 & RL12 & RL13 & RL21 & RL22 & RL23 \\
\hline 1 & $\mathbf{1 . 0 0}$ & $\mathbf{1 . 0 0}$ & $\mathbf{1 . 0 0}$ & $\mathbf{1 . 0 0}$ & $\mathbf{1 . 0 0}$ & $\mathbf{1 . 0 0}$ \\
\hline 10 & $\mathbf{0 . 9 8}$ & $\mathbf{0 . 9 9}$ & $\mathbf{0 . 9 8}$ & $\mathbf{0 . 9 7}$ & $\mathbf{0 . 9 7}$ & $\mathbf{0 . 9 9}$ \\
\hline 20 & $\mathbf{0 . 7 8}$ & $\mathbf{0 . 7 5}$ & $\mathbf{0 . 8 7}$ & $\mathbf{0 . 7 7}$ & $\mathbf{0 . 8 6}$ & $\mathbf{0 . 6 1}$ \\
\hline 30 & $\mathbf{0 . 7 4}$ & $\mathbf{0 . 7 8}$ & 0.51 & 0.38 & $\mathbf{0 . 7 5}$ & 0.55 \\
\hline 40 & $\mathbf{0 . 7 4}$ & $\mathbf{0 . 7 5}$ & 0.23 & $\mathbf{0 . 7 5}$ & 0.64 & $\mathbf{0 . 8 8}$ \\
\hline 50 & $\mathbf{0 . 7 9}$ & $\mathbf{0 . 7 5}$ & 0.39 & 0.60 & 0.54 & 0.67 \\
\hline 60 & $\mathbf{0 . 7 3}$ & $\mathbf{0 . 8 8}$ & 0.40 & $\mathbf{0 . 7 1}$ & $\mathbf{0 . 7 4}$ & 0.49 \\
\hline 70 & 0.36 & $\mathbf{0 . 8 3}$ & 0.60 & 0.10 & 0.64 & $\mathbf{0 . 7 0}$ \\
\hline 80 & $\mathbf{0 . 7 7}$ & $\mathbf{0 . 9 2}$ & 0.38 & 0.49 & 0.46 & 0.45 \\
\hline 90 & 0.59 & 0.61 & 0.42 & $\mathbf{0 . 7 3}$ & -0.01 & 0.53 \\
\hline 100 & 0.37 & $\mathbf{0 . 8 9}$ & 0.13 & 0.42 & 0.47 & 0.55 \\
\hline
\end{tabular}

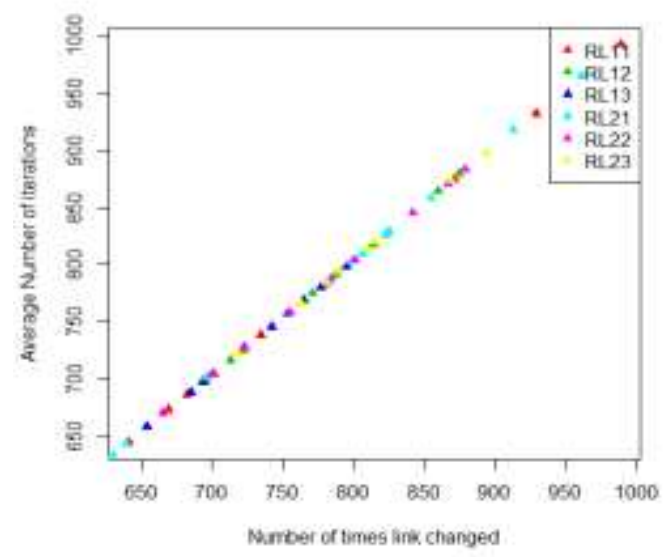

Fig. 12 Average number of iterations vs Number of times link changed for Step Function D10 with link change threshold=1 


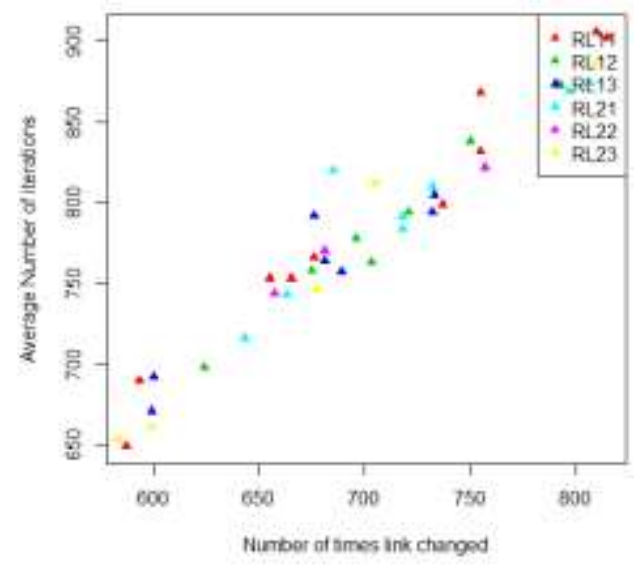

Fig.13. Average number of iterations vs Number of times link changed for Sphere Function D10 with link change threshold=10

Correlation is calculated using "Pearson" method for Link changes and all variants. Tables 14, 15, 16 17, 18 and 19 show correlation calculated using "Pearson" method. Bold faced values represent perfect or strong correlation.

NA: Not Applicable (As number of times link changed during progress is 0 for some threshold values, So if all values are same then the mean value is same and there is no deviation obviously).

Table 20. Correlation Observations

\begin{tabular}{|c|c|c|c|c|c|c|}
\hline \multirow{2}{*}{$\begin{array}{c}\text { Link } \\
\text { change } \\
\text { threshold }\end{array}$} & \multicolumn{3}{|c|}{ Sphere } & \multicolumn{3}{c|}{ Rastrigin } \\
\cline { 2 - 7 } & perfect & strong & weak & perfect & strong & weak \\
\hline 1 & 6 & 0 & 0 & 6 & 0 & 0 \\
\hline 10 & 0 & 6 & 0 & 6 & 0 & 0 \\
\hline 20 & 0 & 2 & 4 & 3 & 3 & 0 \\
\hline 30 & 0 & 0 & 6 & 2 & 4 & 0 \\
\hline 40 & 0 & 2 & 4 & 0 & 6 & 0 \\
\hline 50 & 0 & 1 & 5 & 0 & 6 & 0 \\
\hline 60 & 0 & 0 & 6 & 0 & 6 & 0 \\
\hline 70 & 0 & 0 & 6 & 0 & 5 & 1 \\
\hline 80 & 0 & 0 & 4 & 0 & 4 & 2 \\
\hline 90 & 0 & 0 & 5 & 0 & 4 & 2 \\
\hline 100 & 0 & 0 & 3 & 0 & 5 & 1 \\
\hline
\end{tabular}

Table 21. Correlation Observations

\begin{tabular}{|c|c|c|c|c|c|c|}
\hline \multirow{2}{*}{$\begin{array}{c}\text { Link } \\
\text { change } \\
\text { threshold }\end{array}$} & \multicolumn{3}{|c|}{ Step } & \multicolumn{3}{c|}{ Rosenbrock } \\
\cline { 2 - 7 } & perfect & Strong & weak & perfect & strong & weak \\
\hline 1 & 6 & 0 & 0 & 6 & 0 & 0 \\
\hline 10 & 0 & 6 & 0 & 5 & 1 & 0 \\
\hline 20 & 0 & 4 & 2 & 2 & 4 & 0 \\
\hline 30 & 0 & 4 & 1 & 0 & 6 & 0 \\
\hline 40 & 0 & 2 & 1 & 0 & 6 & 0 \\
\hline 50 & 0 & 1 & 0 & 0 & 6 & 0 \\
\hline 60 & 0 & 0 & 0 & 0 & 2 & 4 \\
\hline 70 & 0 & 0 & 0 & 0 & 3 & 3 \\
\hline 80 & 0 & 0 & 0 & 0 & 2 & 4 \\
\hline 90 & 0 & 0 & 0 & 0 & 3 & 3 \\
\hline 100 & 0 & 0 & 0 & 0 & 1 & 5 \\
\hline
\end{tabular}

If the link is updated after unsuccessful iteration then number of iterations required versus number of times link changed has perfect correlation over runs. Other findings are listed in table 20 and 21.

\section{CONCLUSION}

Particle Swarm Optimization is swarm or population based optimization technique. To handle position and velocity confinement, different bound handling methods are used. Fixed pattern for average number of iterations required for type of bound handling methods and link change variations is not observed. But the standard deviation is more when link is changed after 30 to 50 unsuccessful iterations. Adaptive random neighborhood PSO with varying link structure after threshold number of unsuccessful iterations is tested. The experiments carried out using benchmark functions comprising of unimodal, multimodal, separable and non separable functions. The perfect positive correlation is observed in all variations for Adaptive random neighborhood PSO with varying link structure after unsuccessful iteration while the strong positive correlation is observed in all variations for Adaptive random neighborhood PSO with varying link structure after ten unsuccessful iterations. When the threshold number of unsuccessful iterations increases, correlation value moves towards weak.

\section{ACKNOWLEDGMENT}

The authors wish to thank the Principal and Research Coordinator of Padamashree Dr. D. Y. Patil Institute of Engineering and Technology, Pune.

\section{REFERENCES}

[1] J. Kennedy, R.C. Eberhart, "Particle swarm optimization," Proceedings of the IEEE International Conference on Neural Networks, Piscataway, NJ, USA, 1995, Vol. 4, pp. $1942-1948$

[2] R.C. Eberhart, J. Kennedy, "A new optimizer using particle swarm theory", Proceedings of the Sixth International Symposium on Micro Machine and Human Science, Nagoya, Japan, 1995, pp. $39-43$

[3] J. Kennedy, R. C. Eberhart, and Y. H. Shi, Swarm Intelligence.San Mateo, CA: Morgan Kaufmann, 2001.

[4] Maurice Clerc, "A method to improve Standard PSO," Tech. Rep. MC2009, pp. 03-13, 2009, http://clerc.maurice.free.fr/pso/Design efficient PSO.pdf

[5] Maurice Clerc, "Standard Particle Swarm Optimisation," Particle Swarm Central, Tech. Rep., 2012, http://clerc.maurice.free.fr/pso/SPSO descriptions. pdf

[6] Maurice Clerc, "Beyond standard particle swarm optimisation," International Journal of Swarm Intelligence Research, vol. 1, no. 4, pp. 46-61, 2010.

[7] Zambrano-Bigiarini, M. Clerc, Rojas, "Standard Particle Swarm Optimisation 2011 at CEC-2013: A baseline for future PSO improvements". IEEE Congress on Evolutionary Computation (CEC) 2013 ,Digital Object Identifier: 10.1109/CEC.2013.6557848, Page(s): 2337 2344 
[8] PSC, "Particle Swarm Central," 2013, http://www.particleswarm.info/.

[9] Momin Jamil and Xin-She Yang, A literature survey of benchmark functions for global optimization problems, Int. Journal of Mathematical Modelling and Numerical Optimisation \}, Vol. 4, No. 2, pp. 150--194 (2013)

[10] Christopher K. Monson and Kevin D. Seppi. Exposing Origin-Seeking Bias in PSO. In GECCO'05, pages 241_248, Washington, DC, USA, 2005.

[11] William M. Spears, Derek T. Green, and Diana F. Spears. Biases in particle swarm optimization. International Journal of Swarm Intelligence Research, 1(2):34_57, 2010

[12] Yuhui Shi , Eberhart, R, "A modified particle swarm optimizer “, Evolutionary Computation Proceedings, 1998. IEEE World Congress on Computational Intelligence, 4-9 May 1998 PP $69-73$

[13] R. Poli, J. Kennedy, and T. Blackwell. Particle swarm optimization. Swarm Intelligence, 1(1), 2007, pp. 33-57

[14] Y. Shi, and R.C. Eberhart, "Empirical study of particle swarm optimization", Proceedings of the 1999 Congress on Evolutionary Computation, pp. $1945-1950$

[15] Y. Zheng, et. al., "Empirical study of particle swarm optimizer with an increasing inertia weight", Proceeding of the IEEE Congress on Evolutionary Computation, 2003

[16] Huailiang Liu, Ruijuan Su, Ying Gao , Ruoning Xu, Improved Particle Swarm Optimization Using Two Novel Parallel Inertia Weights, Second International Conference on Intelligent Computation Technology and Automation, IEEE 2009pp 185-188

[17] A. Adriansyah, and S.H.M. Amin, "Analytical and empirical study of particle swarm optimization with a sigmoid decreasing inertia weight", Regional Conference on Engineering and Science, Johor, 2006

[18] A.Nikabadi, M.Ebadzadeh , "Particle swarm optimization algorithms with adaptive Inertia Weight : A survey of the state of the art and a Novel method", IEEE journal of evolutionary computation, 2008

[19] Gao Yue-lin, Duan Yu-hong, "A New Particle Swarm Optimization Algorithm with Random Inertia Weight and Evolution Strategy" International Conference on Computational Intelligence and Security Workshops, IEEE 2007

[20] Y. Feng, G.F. Teng, A.X. Wang, and Y.M. Yao., "Chaotic Inertia Weight in Particle Swarm Optimization", In Innovative Computing, Information and Control, 2007. ICICIC'07. Second International Conference on, page 475. IEEE, 2008

[21] K. Kentzoglanakis and M. Poole., "Particle swarm optimization with an oscillating Inertia Weight", In Proceedings of the 11th Annual conference on Genetic and evolutionary computation, pages 1749-1750. ACM, 2009

[22] Y. Gao, X. An, and J. Liu., "A Particle Swarm Optimization Algorithm with Logarithm Decreasing Inertia Weight and Chaos Mutation", In Computational Intelligence and Security, 2008. CIS'08. International Conference on, volume 1,IEEE, 2008, pp. 61-65

[23] G. Chen, X. Huang, J. Jia, and Z. Min., Natural exponential Inertia Weight strategy in particle swarm optimization, In Intelligent Control and Automation, 2006. WCICA 2006. The Sixth World Congress on, volume 1, pages 3672-3675. IEEE, 2006

[24] Hui-rong Li, Yue-lin Gao, Particle Swarm Optimization Algorithm with Adaptive Threshold Mutation, in the proceeding of International Conference on Computational Intelligence and Security, Beijing, China, December 11December 14 2009, ISBN: 978-0-7695-3931-7,pp. 129-132
[25] H.R. Li and Y.L. Gao., Particle Swarm Optimization Algorithm with Exponent Decreasing Inertia Weight and Stochastic Mutation, In -2009 Second International Conference on Information and Computing Science,IEEE, 2009, pp 66-69

[26] Shi Y. Eberhart RC, Fuzzy adaptive particle swarm optimization, Evolutionary Computation, 2001. Proceedings of the 2001 Congress, Volume 1, Issue, 2001, pp. $101-106$

[27] J. Kennedy and R. Mendes, Population structure and particle swarm performance, in Proc. 2002 Cong. Evol. Comput., 2002, pp.1671-1675

[28] Rui Mendes, James Kennedy, and José Neves, The Fully Informed Particle Swarm: Simpler, Maybe Better, IEEE Transactions On Evolutionary Computation, Vol. 8, No. 3, June 2004

[29] Helwig S, Branke, J. ,Mostaghim, S.M., "Experimental Analysis of Bound Handling Techniques in Particle Swarm Optimization", Evolutionary Computation, IEEE Transactions on (Volume:17, Issue: 2 ), April 2013, pp. 259-271

[30] Maurice clerc, Particle Swarm Optimization, ISTE ( International Scientific and Technical Encyclopedia, 2006

[31] J. Kennedy and R. Mendes, Population structure and particle swarm performance, in Proc. 2002 Cong. Evolutionary Computation, 2002, pp.1671-1675

\section{Authors' Profiles}

Mrs. Snehal Kamalapur received ME (Computer Engineering) degrees from University of Pune 2003. She is working as associate professor in the $\mathrm{K} \mathrm{K}$ Wagh Institute of Engineering Education and Research, University of Pune. She is currently pursuing PhD from university of Pune. She has published over 28 research papers in conferences and Journals. She is life member of various professional bodies. Her research interests include swarm intelligence, evolutionary computation and pattern recognition.

Dr. Varsha Patil is doctorate in Computer Engineering. She is working as Professor with additional reponsibility as Vice Principal in Matoshri College of Engineering \& Research Centre, Nashik. She is member of board of studies of Computer Engineering at University of Pune. She has authored 2 books titled 'Discrete Mathematics' \& 'Data Structures in C++' published by McGraw Hill \& Oxford University Press. She has 40 papers to her credit, which are published at various national and international Journals and conferences. She is life member of various professional bodies. Her research interest includes Image processing, Evolutionary computation, Parallel Computing and Swarm Intelligence.

How to cite this paper: Snehal Mohan Kamalapur, Varsha Hemant Patil,"Adaptive Random Link PSO with Link Change Variations and Confinement Handling", International Journal of Intelligent Systems and Applications (IJISA), vol.7, no.1, pp.62-72, 2015. DOI: 10.5815/ijisa.2015.01.06 